Bangladesh J. Plant Taxon. 27(2): 453-459, 2020 (December)

(C) 2020 Bangladesh Association of Plant Taxonomists

\title{
FIRST RECORD OF LEUCOAGARICUS NIVALIS FROM PAKISTAN
}

\author{
Sana Jabeen*, Bushra Waseem, Tuba, Mughees Hamid \\ and ANeela Yasmeen ${ }^{1}$ \\ Department of Botany, Division of Science and Technology, University of Education, \\ Township, Lahore, Punjab, Pakistan
}

Keywords: Agaricoid; Changa Manga; Taxonomy.

Leucoagaricus Locq. ex Singer is represented by more than 150 species of agaricoid, saprotrophic fungi distributed all over the world (Kirk et al., 2008; Kumari and Atri, 2013; Yuan and Liang, 2014; Nabe et al., 2014; Ge et al., 2017; Justo et al., 2015; Qasim et al., 2015; Yu et al., 2016; Hussain et al., 2018; Usman and Khalid 2018; Verma and Vimal, 2018; Sysouphanthong et al., 2018; Yang et al., 2019; Ullah et al., 2020). Only 11 Leucoagaricus species have been reported from Pakistan so far (Ahmad et al., 1997; Qasim et al., 2015; Ge et al., 2017; Hussain et al., 2018; Usman and Khalid, 2018; Ullah et al., 2020). Leucoagaricus is characterized by small to medium, fleshy or thin basidiomata, ranging in stature from slender to sturdy; a pileus surface that is radially fibrillose, floccose, squamulose to fibrillose-scaly or granulose (rarely); entire or very short striate margins; a central, equal to bulbous stipe with a membranous, sometimes moveable annulus; metachromatic basidiospores generally lack a welldefined germ pore and are thin-walled and smooth; and the pileipellis is either a trichoderm or a cutis of repent and radially arranged hyphae lacking sphaerocysts. Pleurocystidia are absent in most species. Clamp connections are absent (Singer 1986; Vellinga 2001).

The present study focuses on morphological and molecular characterization of a Leucoagaricus species collected in the Changa Manga forest, Kasur district, Punjab, Pakistan. This research is an effort to establish the fungal diversity of this forest. During field survey in 2019 for the collection of macrofungi to explore the diversity of these fungi from Changa Manga. A number of basidiomata of Leucoagaricus were collected. Field notes were recorded and the samples were air dried and preserved for future analysis. Macroscopic descriptions were based on the fresh material. Significant characters involve size, shape and color of the pileus; attachment and color of lamellae; presence of annulus on stipe. Color codes were given using Munsell (1975) color system. For micro-morphology, dried samples were examined using standard microscopic techniques. Different chemicals were used as mounting media according to requirements. For rehydration, 5\% $\mathrm{KOH}$ was used, and for staining the walls of hyaline hyphae, Congo red was used. The anatomical features were observed under microscope Xsz 107BN adjusting at 100x objective lens. Measurements were noted using calibrated Motic Images Plus 2.0 software. For basidiospores, $[\mathrm{n} / \mathrm{m} / \mathrm{p}]$ represents $\mathrm{n}$ number of spores, measured from $\mathrm{m}$ basidiomata and $\mathrm{p}$ collections, $1 \times \mathrm{w}$ represents spore dimensions, extreme values are given in parenthesis. $\mathrm{Q}$ values are given as $1 \times \mathrm{w}$ ratio while definitions of the $\mathrm{Q}$ values for spores are given following Bas (1969). Drawings were made from the laptop screen. The examined specimens are deposited in the herbarium (LAH), Department of Botany, University of the Punjab, Quaid-e-Azam Campus, Lahore, Pakistan. For DNA extraction, the Extract-N-Amp ${ }^{\mathrm{TM}}$ kit (Sigma- Aldrich, St Louis, MO, USA) was used following the manufacturer's protocol. PCR amplification and sequencing was carried

*Corresponding author, E-mail: sanajabeenue@gmail.com; sanajabeen@ue.edu.pk

${ }^{1}$ Centre for Excellence in Molecular Biology, University of the Punjab, 87-West Canal Bank Road, Near Thokar Niaz Baig, Lahore, Punjab, Pakistan. 
out from the sequence service using ITS1F and ITS4 primers. Sequences obtained were analyzed in BioEdit sequence alignment editor version 7.2.5 (Hall, 1999). Consensus sequences were generated and BLAST searched at NCBI (http://www.ncbi.nlm.nih.gov/). Sequences with closest match were selected from GenBank to reconstruct phylogeny. The sequences with incomplete ITS region were left aside. Published sequences of the closest relatives of the species were included to reconstruct phylogeny (Hussain et al., 2018; Ullah et al., 2020). Agaricus bisporus (J.E. Lange) Imbach (AF432886) and Agaricus campestris L. (U85307) were chosen as outgroup to root the phylogenetic tree. The sequences were aligned using an online MUSCLE tool at EMBL-EBI (http://www.ebi.ac.uk/). A maximum likelihood tree was inferred using Tamura 3-parameter model (Tamura, 1992) by best DNA model selection in MEGA 6 (Tamura et al., 2013). The percentage of trees in which the associated taxa clustered together is shown next to the branches. The tree is drawn to scale, with branch lengths measured in the number of substitutions per site. The analysis involved a selection of 117 nucleotide sequences. There were a total of 866 positions in the final dataset. The phylogeny was tasted with 1000 bootstrap replicates.

BLAST at NCBI revealed that the complete ITS sequences from Pakistani collections of Leucoagaricus showed 99-100\% similarity with the sequences from China (KY039573) and Pakistan (MK106150-MK106153). These sequences along with other sequences from closely related taxa constituted a final dataset of 117 nucleotide sequences with 866 positions. Among these positions, 296 were conserved, 540 were variable, 436 were parsimony informative and 90 were represented as singletons. The phylogenetic tree recovered from this dataset is shown in Fig. 1. The sequences from our collections were clustered within a clade including sequences of $L$. nivalis (W.F. Chiu) Z.W. Ge \& Zhu L. Yang, L. purpureolilacinus Huijsman, L. umbonatus Hussain et al., and some unidentified taxa. Our sequences clustered within the L. nivalis lineage including sequences from Changa Manga forest Pakistan and China with 100\% bootstrap value Fig. 1.

Taxonomy:

Leucoagaricus nivalis (W.F. Chiu) Z.W. Ge \& Zhu L. Yang Mycosystema 36(5): 548 (2017)

(Fig. 2 \& 3)

Pileus $2.3-7.5 \mathrm{~cm}$, broadly convex to flat becoming uplifted when mature, surface smooth to slightly fibrillose, white, slightly umbonate; umbo light yellow (2.5Y9/4), margin striate, undulating to dentate or eroded. Lamellae free, crowded, narrow to ventrocose, entire, white. Lamellulae frequent, of variable lengths. Stipe $(1.1-) 2-4(-11) \times(0.2-) 0.3-0.5(-0.6) \mathrm{cm}$, with upto $0.8 \mathrm{~cm}$ wide base, slightly narrow towards the pileus, central, cylindrical, sometimes curved, smooth, white. Annulus present, inferior, white (2.5Y8/4). smell and taste not observed. Basidiospores [60/3/3] (7.8-)8.3-12(-12.6) $\times(5.7-) 6.3-7.7(-8) \mu \mathrm{m}, \mathrm{Q}=(1.3-) 1.5-1.7(-2)$, avQ $=$ 1.5, ellipsoid, amygdaliform in side view, ovoid in front view; dextrinoid; apiculus prominent; germ pore absent. Basidia (13.8-)13.9-15.5(-17.2) $\times(5.4-) 5.5-6.4(-7.2) \mu \mathrm{m}$, clavate, with $2-4$ sterigmata. Lamellae edge sterile Cheilocystidia (11-)14-14.9(-16.1) $\times(4.2-) 4.8-4.9(-5) \mu \mathrm{m}$, clavate. Pleurocystidia absent. Pileipellis hyphae (5-)5.4-8.1(-8.8) $\mu \mathrm{m}$ wide, septate; septa frequent; hyphal terminals at the center of the pileus cylindrical (5.2-)5.4-6(-6.6) $\mu \mathrm{m}$ wide. Stipitipellis hyphae (6.2-)9.4-10.8(-12.4) $\mu \mathrm{m}$ wide, septate; septa frequent. Clamp connections absent in all tissues. All hyphal walls are transparent in $\mathrm{H}_{2} \mathrm{O}$ and pink in Congo red.

Specimen examined: PAKISTAN. Punjab: Lahore division, Kasur district, Changa Manga, $192 \mathrm{~m}$ a.s.l., on soil 6 October 2019, Sana Jabeen SJ51CM4 (LAH36651; GenBank: MT573439); SJ53CM5 (LAH36652; GenBank: MT573440); Tuba SJ58CM12 (LAH36653; GenBank: MT573441). 


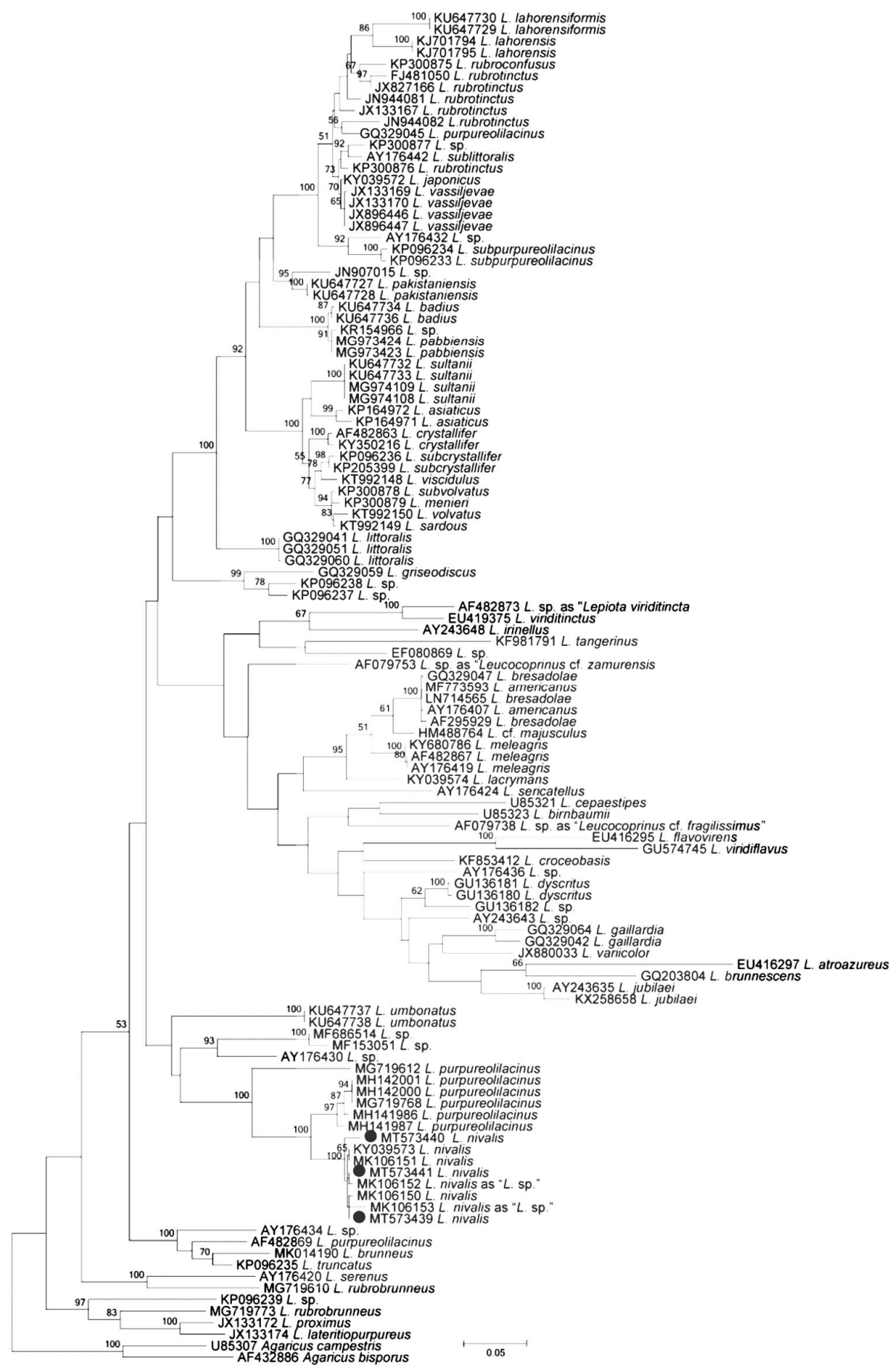

Fig. 1. Molecular phylogenetic analysis of Leucoagaricus spp. based on ITS sequences. Sequence generated during this study are marked by $\bullet$. Scale bar = nucleotide substitutions per site. 


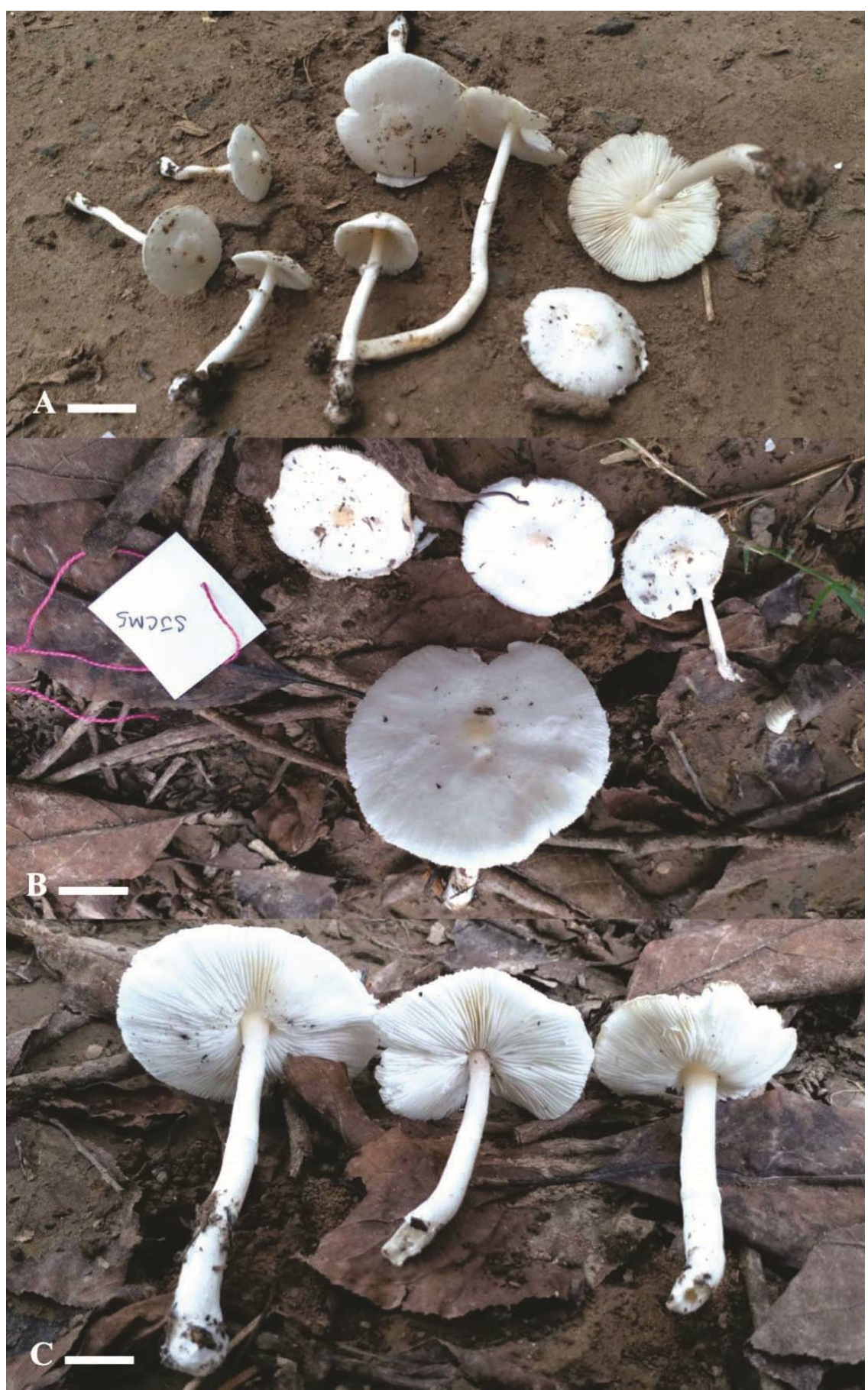

Fig. 2. Leucoagaricus nivalis basidiomata. A. LAH36651; B \& C. LAH36652. Scale bars $=2 \mathrm{~cm}$. Photos by Sana Jabeen. 


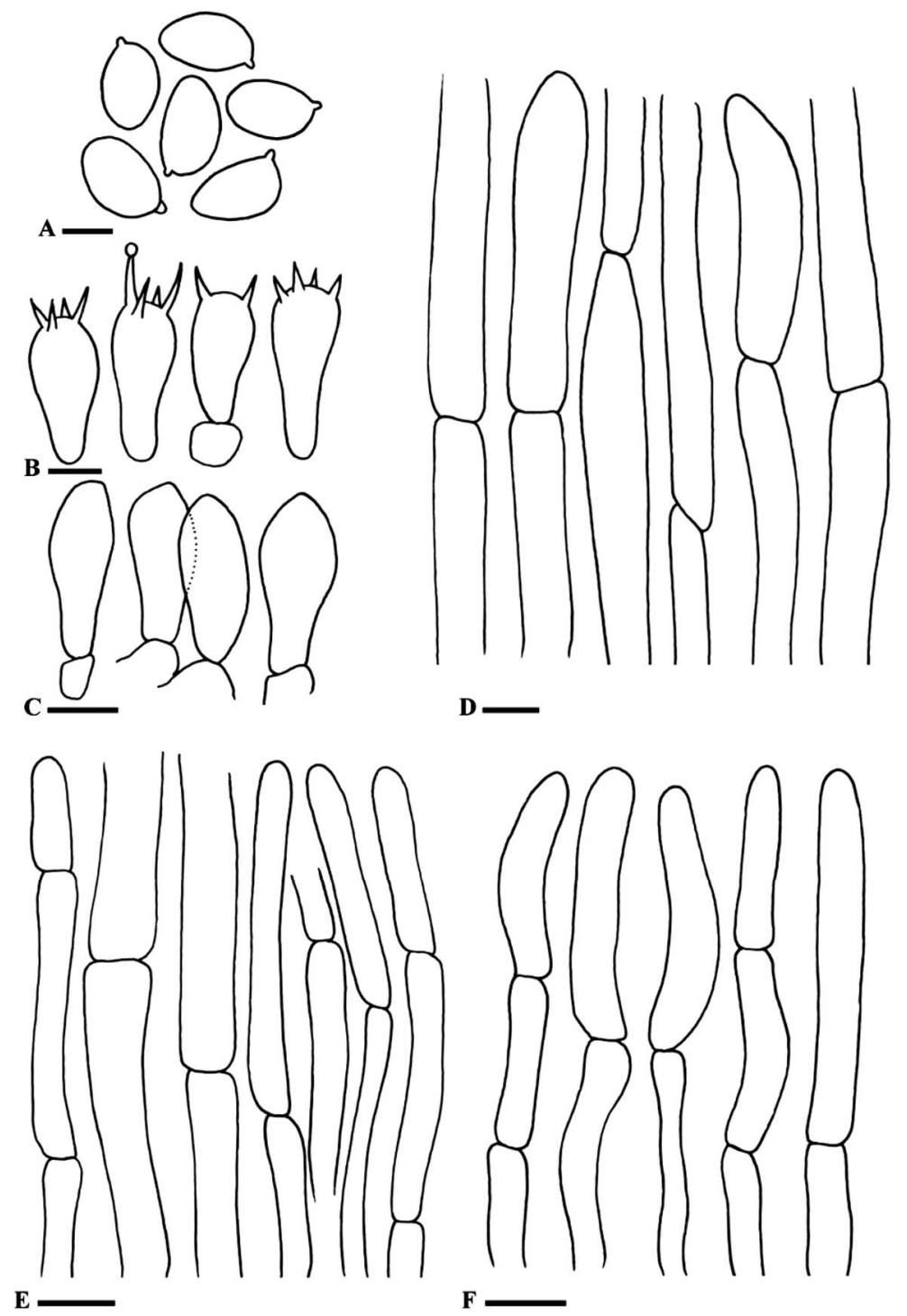

Fig. 3. Leucoagaricus nivalis (LAH36652). A. Basidiospores; B. Basidia; C. Cheilocystidia; D. Stipitipellis; E. Pileipellis; F. Pileipellis terminal hyphae from umbo. Scale bars: A-C $=5 \mu \mathrm{m}, \mathrm{D}-\mathrm{F}=10 \mu \mathrm{m}$. Drawings by Sana Jabeen.

Comments: Leucoagaricus nivalis was described in 1948 as Lepiota nivalis W.F. Chiu from Kunming, Yunnan province, China (Chiu, 1948). Recent studies by Yang and Ge (2017) revealed that this species belongs to Leucoagaricus based on its features of basidiospores and cheilocystidia. Leucoagaricus nivalis was morphologically identified based on the type collection and two modern collections from the same area. Our collections showed more or less similar features to the type collection of L. nivalis HMAS 4237 (Yang and Ge, 2017). Though there are some minor differences that were observed in comparative study. These features include the size of the basidiomata which is smaller $(6-7.5 \times 3.5-4.5)$ and the size of the basidiospores in terms of 
Q value that is slightly larger (1.7) in Chinese collections. Leucoagaricus nivalis has only been reported from China (Chiu, 1948; Yang and Ge, 2017). Already available sequences in GenBank belong to collections from Changa Manga, Pakistan (MK106150-MK106153) and modern Chinese collection from Yunnan province (KY039573). In phylogenetic tree, the sequences generated during this study clustered with these sequences in the same clade supports its taxonomy as L. nivalis. Leucoagaricus nivalis has only been validly published from China (Chiu, 1948; Yang and Ge, 2017), occurrence of L. nivalis in Pakistan is an addition to the funga of Pakistan.

\section{Acknowledgements}

Sincere thanks to Dr. Arun Kumar Thiruvoth Kottuvetta (The Zamorin's Guruvayurappan College, Guruvayurappan College, Kozhikode, Kerala 673014, India) and Dr. Else C. Vellinga (University of California, Berkeley, California, USA) for presubmission review of the manuscript. Their comments and suggestions greatly helped to improve the document. We are thankful to Dr. Kiran Yasmeen Malik, Mr. Amir Ali and Mr. Muhammad Kamran for their help in sampling.

\section{References}

Ahmad, S., Iqbal, S. H. and Khalid, A. N. 1997. Fungi of Pakistan. Sultan Ahmad Mycological Society Pakistan pp. 1-248. https://doi.org/10.3852/14-351

Bas, C. 1969. Morphology and subdivision of Amanita and a monograph of its section Lepidella. Persoonia 5: $285-579$.

Chiu, W. F.1948. The Amanitaceae of Yunnan. The Science Reports of National TsingHua University. Series B, Biological and Psychological Sciences 3(3): 165-178.

Ge, Z. W., Yang, Z. L., Qasim, T., Nawaz, R., Khalid, A. N. and Vellinga, E. C. 2017. Four new species in Leucoagaricus (Agaricaceae, Basidiomycota) from Asia. Mycologia 107(5): 1033-1044. https://doi.org/ $10.3852 / 14-351$

Hall, T. A. 1999. BioEdit: a user-friendly biological sequence alignment editor and analysis program for Windows 95/98/NT. In Nucleic Acids Symposium Series 41(41): 95-98.

Hussain, S., Jabeen, S., Khalid, A. N., Ahmad, H., Afshan, N. S., Sher, H. and Pfister, D. H. 2018. Underexplored regions of Pakistan yield five new species of Leucoagaricus. Mycologia 110 (2): $387-$ 400. https://doi.org/10.1080/00275514.2018.1439651

Justo, A., Angelini, C., Bizzi, A. and Vizzini, A. 2015. Leucoagaricus sabinae (Agaricaceae), a new species from the Dominican Republic. North American Fungi 10: 1-15. https://doi.org/10.11646/phytotaxa.226.1.9

Kirk, P. M., Cannon, P. F., Minter, D. W. and Stalpers, J. A. eds. 2008. Dictionary of Fungi, 10 ${ }^{\text {th }}$ edn.CABI, Wallingford.

Kumari, B. and Atri, N.S. 2013. New additions of basidiomycetous fungi in Indian mycoflora. Mycosphere 4(1): 53-59. https://doi.org/10.5943/mycosphere/4/1/4

Munsell, A. H. 1975. Munsell soil color charts. Baltimore, MD, USA.

Nabe, M., Kasya, T. and Hosaka K. 2014. Leucoagaricus viridiflavus (Agaricaceae), new to Japan. Japanese Journal of Mycology 55(2): 35-40.

Qasim, T., Amir, T., Nawaz, R., Niazi, A. R. and Khalid, A. N. 2015. Leucoagaricus lahorensis, a new species of L. sect. Rubrotincti. Mycotaxon 130(2): 533-541. https://doi.org/10.5248/130.533

Singer, R. 1986. The Agaricales in modern taxonomy, $4^{\text {th }}$ edn. Koeltz Scientific Books , Koenigstein.

Sysouphanthong, P., Bouamanivong, S., Salichanh, T., Xaybouangeun, N., Sucharitakul, P., Osathanunkul, M. and Suwannapoom, C. 2018. Leucoagaricus houaynhangensis (Agaricaceae), A new yellowishgreen species from Lao People's Democratic Republic. Chiang Mai Journal of Science 45(3): 12871295. 
Tamura, K., Stecher, G., Peterson, D., Filipski, A. and Kumar, S. 2013. MEGA6: Molecular evolutionary genetics analysis version 6.0. Molecular Biology and Evolution 30: 2725-2729. https://doi.org/10.1093/ molbev/mst197

Tamura, K. 1992. Estimation of the number of nucleotide substitutions when there are strong transitiontransversion and G + C-content biases. Molecular Biology and Evolution 9: 678-687.

Ullah, Z., Jabeen, S., Faisal, M., Ahmad, H. and Khalid, A. N. 2020. Leucoagaricus brunneus sp. nov. from Khyber Pakhtunkhwa, Pakistan. Mycotaxon 134(4): 601-611. https://doi.org/10.5248/134.601

Usman, M. and Khalid, A. N. 2018. Leucoagaricus pabbiensis sp. nov. from Punjab, Pakistan. Mycotaxon 133(2): 354-363. https://doi.org/10.5248/133.355

Vellinga, E. C. 2001. Leucoagaricus. In: Noordeloos, M.E., Kuyper, T.W. and Vellinga, E.C. (Eds.). Flora Agaricina Neerlandica 5. Rotterdam: 85-108.

Verma, R. K. and Vimal, P. H. L. A. 2018. Diversity of macro-fungi in Central India-XII: Leucoagaricus rubrotinctus. Van Sangyan 5(4): 1-10.

Yang, Z. L., Ge, Z. W. and Liang J. 2019. Flora Fungorum sinicorum vol. 52. Fungi Lepiotoidei (Agaricaceae).

Yang, Z. L and Ge, Z. W. 2017. Six new combinations of lepiotaceous fungi from China. Mycosystema 36(5): 542-551.

Yu, F., Liang, J. F., Ge, Z. W. and Li, Y. K. 2016. Morphological and molecular evidence for a new species of Leucoagaricus from China. Sydowia 68: 41-47.

Yuan Y, Li, Y. K. and Liang, J. F. 2014. Leucoagaricus tangerinus, a new species with drops from Southern China. Mycological Progress 13(3): 893-898. https://doi.org/10.1007/s11557-014-0974-2

(Manuscript received on 25 August, 2020; revised on 19 November, 2020) 